\title{
УПРАВЛЕНИЕ ИНФОРМАЦИОННЫМИ ТЕХНОЛОГИЯМИ КАК СОСТАВЛЯЮЩАЯ СРМ-СИСТЕМ
}

\section{INFORMATION TECHNOLOGY MANAGEMENT AS A COMPONENT OF CPM-SYSTEMS}

\section{Odintsova}

Summary. The article raises the question of the possibilities of automating the information technology (IT) management process in accordance with modern standards and practices. Based on the completed review of modern standards, methods and practices of IT management, it was concluded that it is necessary to coordinate the goals and objectives of the development of IT infrastructure components with the strategic plans of the enterprise. The functionality and architecture of CPM-systems as a class of software products for automating enterprise management at all levels are considered. Analysis of the possibilities for automation of information technology management based on CPM-systems allows us to talk about the prospects for the development of information technologies in this direction.

Keywords: information technology management, IT, Performance Management, CPM-systems, ITIL, COBIT, ITOM.

\section{Ввемение}

$\mathbf{y}$ правление информационными технологиями является важной составляющей успешного развития современных предприятий и повышения эффективности их функционирования. Хорошо организованная, грамотно спроектированная, с учетом особенностей деятельности компании, ИТ-инфраструктура является основой для стабильной работы и достижения поставленных целей предприятием. Необходимость управления информационными технологиями отмечают специалисты на проводимых конференциях [10] и форумах, об этом свидетельствует и повышающийся интерес бизнеса к услугам, связанным с поддержкой и обслуживанием ИТ-инфраструктуры, разработкой ИТ-стратегии.

На отечественном рынке представлены программные продукты для автоматизации деятельности ИТ-подразделений компаний, служб технической поддержки. Такие информационные системы, как правило, поддерживают процесс управления ИТ на оперативном уровне. Достаточно ли только их использования для комплексного и полномасштабного управления ИТ? Для ответа
Одинцова Мария Александровна

К.э.н., доцент, РТУ МИРЭА (г. Москва) maryod@rambler.ru

Аннотация. В статье ставится вопрос о возможностях автоматизации процесса управления информационными технологиями (ИТ) согласно современным стандартам и практикам. На основании выполненного обзора современных стандартов, методик и практик управления ИТ сделан вывод о необходимости согласования целей и задач развития компонентов ИТ-инфраструктуры со стратегическими планами предприятия. Рассмотрены функциональные возможности и архитектура СРМ-систем как класса программных продуктов для автоматизации управления предприятием на всех уровнях. Анализ возможностей автоматизации управления информационными технологиями на базе СРМ-систем позволяет говорить о перспективах развития информационных технологий в этом направлении.

Ключевые слова: управление информационными технологиями, ИТ, управление результативностью, СРМ-системы, ITIL, COBIT, ITOM.

на этот вопрос обратимся к современным стандартам и практикам управления ИТ.

\section{Обзор современных мето ик \\ и по ХХА АВ к управ^ению \\ информационными техноАогиями}

Подходы к управлению информационными технологиями формализованы в определенных стандартах и методиках. Среди наиболее значимых выделяют ГОСТы, международный стандарт TOGAF, библиотеку лучших практик ITIL, COBIT, методологию Дж. А. Захмана.

ГОСТы регламентируют основы описания жизненного цикла систем, процессы и соответствующую терминологию, стадии и этапы разработки автоматизированных информационных систем, а также процессы, работы, операции, выполняемые при приобретении программных продуктов или услуг, их поставке, разработке, эксплуатации, сопровождении и прекращении применения [5-7].

Стандарт TOGAF (The Open Group Architecture Framework) регламентирует архитектуру предприятия, 
определяя слои: бизнес-архитектура, архитектура данных, приложений и технологическая архитектура. Стандарт также определяет подход к разработке архитектуры (ADM), являющийся итеративным и предполагающий разработку целевой архитектуры с учетом взглядов всех заинтересованных сторон, и ее детализацию, начиная с создания бизнес-архитектуры (архитектуры деятельности предприятия в соответствии с целевым видением).

ITIL (Information Technology Infrastructure Library) является одним из наиболее популярных стандартов по управлению ИТ-услугами в соответствии с ITSM (Information Technology Service Management) и ориентирован на согласование потребностей бизнеса с предоставляемыми ему ИТ-сервисами. Предыдущая версия руководства ITIL V.3 была опубликована в виде пяти книг, описывающих стратегию услуг, их проектирование, преобразование, эксплуатацию и непрерывное улучшение. В текущей версии ITIL V.4 представлено два ключевых компонента - система создания ценности услуг и модель четырех измерений (люди, продукты, партнеры, процессы). Одной из ключевых идей новой версии ITIL следует назвать совместное (с бизнесом) создание ценности, а не предоставление ее бизнесу посредством оказания услуг, как это было в V.3.

Актуальная версия COBIT 2019 является логичным продолжением предыдущей версии (СОВІT 52012 г.) и поддерживает основную идею, согласно которой план развития информационных технологий должен базироваться на общекорпоративных целях развития предприятия. Данный стандарт сегодня ассоциируется прежде всего с оценкой зрелости процессов управления информационными технологиями, и СОВІT 2019 предлагает модель для оценки зрелости и развития системы управления информационными технологиями и процессами с учетом, конечно же, потребностей бизнеса и общекорпоративных целей.

Необходимость согласования целей и задач развития информационных технологий с планами развития бизнеса четко прослеживается и в модели Дж. А. Захмана, описывающей архитектуру предприятия и определяющей подход к описанию каждого аспекта предприятия во взаимосвязи с остальными.

Представленный перечень методологий, стандартов и лучших практик не является исчерпывающим, к тому же они не заменяют, а скорее дополняют друг друга. О необходимости совместного использования стандартов, методологий и рекомендаций говорит появившейся в 2014 г. IT4IT — открытый стандарт и референсная архитектура управления ИТ. IT4IT делает акцент не на процессах, а на функциональных компонентах, которые используются в любых компаниях при управ- лении информационными технологиями, независимо от того, какие подходы применяются на разных стадиях и в разных процессах.

В рассмотренных подходах отмечается необходимость согласовывать, а зачастую разрабатывать планы по управлению информационными технологиями в соответствии с целями и задачами развития бизнеса.

Управление информационными технологиями должно осуществляться на стратегическом уровне управления предприятием, «инструмент стратегического управления ИТ» должен являться «частью общей системы менеджмента компании» [2], а ИТ-стратегия должна отражать и поддерживать долгосрочные цели развития бизнеса. Таким образом, отправной точкой при управлении информационными технологиями следуют считать планы развития предприятия.

\section{СРМ-системы как инструмент \\ $\triangle \wedge$ разработки п^анов \\ развития премприятия}

При планировании развития предприятия наиболее популярным подходом сегодня является управление по целям и ключевым показателям эффективности, например, на основе сбалансированной системы показателей, а при организации работы персонала - управление результативностью - Performance Management. Использование сбалансированной системы показателей (ССП) позволяет определить общекорпоративные цели развития бизнеса и на их основе разрабатывать планы развития предприятия, детализируя их в разных временных горизонтах, а также для отдельных видов деятельности, подразделений, команд. Performance Management, дополняя ССП, позволяет эффективно осуществлять управление персоналом на основе поставленных целей и оценивать не только достигнутые результаты, но и соответствие сотрудников определенным требованиям, включая их компетенции, внешний вид и др. При этом специалисты отмечают необходимость не традиционной годовой оценки результатов и работы сотрудников, а постоянной или скользящей (со значительно меньшими лагами) оценки производительности и результатов деятельности — Ongoing Performance Management [14].

На информационно-программном уровне разработку стратегии развития предприятия и настраиваемую оценку результативности деятельности сотрудников поддерживают информационные системы класса CPM (Corporate Performance Management) - системы управления эффективностью организаций. Также известны другие термины: BPM (Business Performance Management), EPM (Enterprise Performance Management), ECM (Enterprise Commerce Management), BAM (Business 


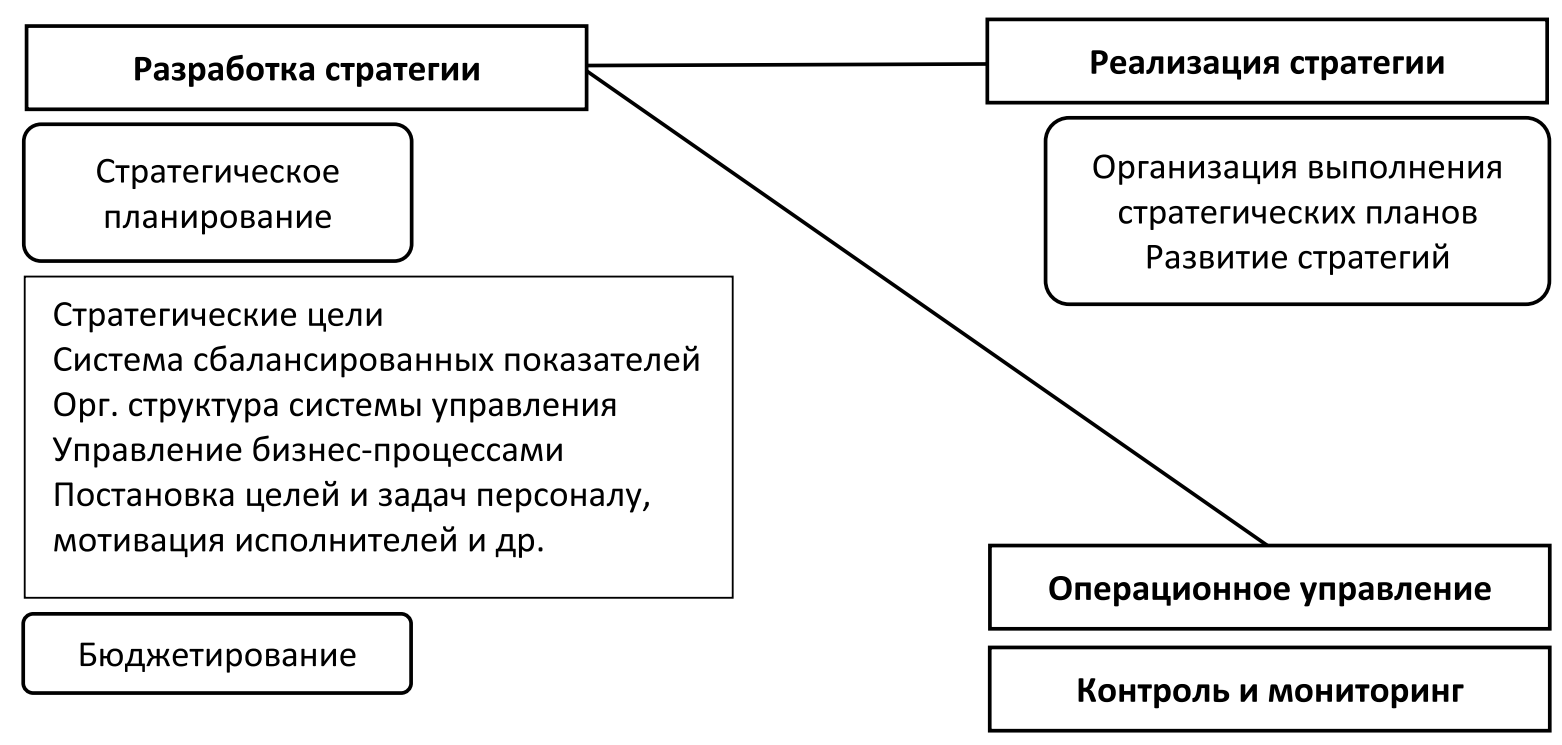

Рис. 1. Поддержка полного цикла управления предприятием СРМ-системами [4]

Activity Management). Система данного класса поддерживают полный цикл управления предприятием, включая поддержку разработки стратегии, ее детализацию при тактическом планировании, постановку целей и задач сотрудникам, контроль и анализ деятельности. На российском рынке известны решения от таких компаний, как «Инталев», «1C», Intersoft Lab, Oracle, SAP, IBM и Adaptive Insights.

По данным исследований, управление результативностью является сегодня одним из востребованных направлений в менеджменте и в разработке информационных систем [3]. Компании, внедряющие управление по целям, показывают более высокие результаты, так как сотрудники ориентированы именно на результат, когда при традиционном подходе - на процесс, как это указано в должностных инструкциях. Еще одной положительной чертой следует считать более тесное взаимодействие руководителей и персонала: при постановке целей и задач исполнителям руководитель часто их обговаривает с подчиненными, благодаря чему задача становится ясной, и сотрудник понимает, что именно он должен сделать, и каким должен быть результат. Информационные системы, позволяют фиксировать все поставленные задачи, расставлять их приоритеты, напоминать о необходимости выполнения и регистрировать достигнутые результаты. При оценке деятельности персонала одной из тенденций является оценка не только достигнутых результатов, но и оценка знаний, навыков и других составляющих, характеризующих сотрудника, например, методом 360 градусов, причем оценивать может не только руководитель, но коллеги, клиенты и другие лица, контактирующие с оцениваемым и пользующиеся результатами его работы.
Системы класса СРМ предоставляют пользователям инструменты, позволяющие:

- формировать консолидированную управленческую, финансовую отчетность за выбранный период;

- осуществлять стратегическое планирование;

- детализировать стратегические планы при создании тактических и оперативных планов;

- поддерживать принятие управленческих решений с помощью анализа накопленных данных и прогнозирования, а также построения оптимизационных и других экономико-математических моделей [13].

Системы класса СРМ позволяют поддерживать полный цикл управления предприятием на всех уровнях (рис. 1).

Поддержка полного цикла управления предприятием осуществляется благодаря наличию в СРМ-системах следующих компонентов:

- подсистема, предназначенная для реализации управления по целям;

- подсистема моделирования бизнеса предоставляет возможности для разработки сценариев работы предприятия с учетом потребляемых ресурсов, выявления узких мест с использованием различных экономико-математических методов, в том числе методов функционально-стоимостного анализа;

- подсистемы бюджетирования, планирования и прогнозирования, необходимые для анализа, поддержки принятия решений и планирования деятельности предприятия; 
- подсистема интеллектуальной бизнес-аналитики, позволяющая консолидировать информацию из различных внутренних и внешних источников, структурировать ее и осуществлять анализ накопленных данных;

- подсистема финансовой и управленческой отчетности, позволяющая агрегировать и визуализировать накопленную информацию, предоставляя руководителям необходимые отчеты в различных разрезах и с различной степенью детализации [9].

- Таким образом, системы класса СРМ имеют все необходимые инструменты, позволяющие полномасштабно осуществлять управление предприятием на всех уровнях.

\section{Примеры систем \\ к^асса СРМ, представленные \\ на отечественном рынке}

По оценкам специалистов [1], российский рынок СРМ-систем соответствует мировым трендам, а отечественные разработки являются качественными конкурентоспособными программными продуктами. При этом растет спрос на подобные информационные системы не только со стороны крупного, но и среднего бизнеса. Компании стремятся задействовать компоненты интеллектуального анализа систем для выявления закономерностей в накопленных данных и поддержки принятия управленческих решений.

Среди отечественных разработок весьма популярными являются решения от компаний «1С» и «Галактика». Рассмотрим их в качестве примеров.

Программный продукт «1С: Управление по целям и КРІ» («ТопФактор») является тиражируемым решением для автоматизации целевого управления на базе «1С: Предприятие». В концепции данного решения управление по целям реализовано на стратегическом, тактическом и оперативном уровнях, а оценка результативности базируется на трех составляющих:

KPI (целевые значения ключевых показателей, например, финансовые и/ или коммерческие показатели);

- задачи (SMART-задачи, например, этапы и вехи проектов, за которые отвечает сотрудник, поручения руководства);

- соответствие определенным требованиям стандартам (данная информация может быть получена на основе оценки руководителя по заранее определенным критериям, оценки коллег, клиентов, соответствия профессиональным стандартам).
Таким образом, регистрируются и оцениваются не только количественные, но и качественные критерии. Далее эти данные сводятся воедино в матрице и выражаются в коэффициенте профессиональной результативности, учитывающем все оцениваемые аспекты. Конечно, данный показатель может быть использован для расчета вознаграждения сотруднику, но он также важен для принятия решений при управлении персоналом. При таком подходе сотрудник понимает, за какие задачи он отвечает, от чего зависит его вознаграждение, а руководитель может своевременно получать информацию о достигнутых результатах и корректировать планы. Система поддерживает формирование рейтингов сотрудников, а также разработку индивидуальных планов развития. Благодаря реализованным в программном продукте маршрутам оценки результативности и планирования, система автоматически выводит напоминания руководителям и сотрудникам о том, когда и какие задачи им необходимо выполнить.

Как и большинство систем данного класса, «1С:Управление по целям и КРІ» является «надстройкой» и требует интеграции с учетными системами, системами бюджетирования для сбора информации и получения данных о достигнутых результатах и их сравнении с целевыми показателями [3].

При сравнении компаний «1С» и «Галактика» обычно отмечают масштаб бизнеса, на автоматизации которого они исторически специализировались. И если фирма «1С» начинала свои проекты с автоматизации малого и среднего бизнеса, то «Галактика» изначально была ориентирована на работу с крупными предприятиями, холдингами.

«Галактика СРМ» сегодня представлена линейкой программных продуктов, включающих в себя решения: «Галактика Бюджетирование», «Галактика Инвестиционное планирование», «Галактика Консолидация отчетности», «Галактика Казначейство», «Галактика В|» и является комплексным решением для автоматизации процессов бюджетирования, финансовой консолидации и формирования консолидированной отчетности предприятия [12]. Концепция решения реализована в двух основных аспектах:

- консолидация отчетности (по международным стандартам финансовой отчётности (МСФО), российским стандартам бухгалтерского учета (РСБУ), внутрикорпоративная и статистическая отчетность);

- планирование, включая бюджетирование, инвестиционное управление, казначейство и др.

Данные в систему попадают из внешних источников, сводных отчетов и транзакционных программных 

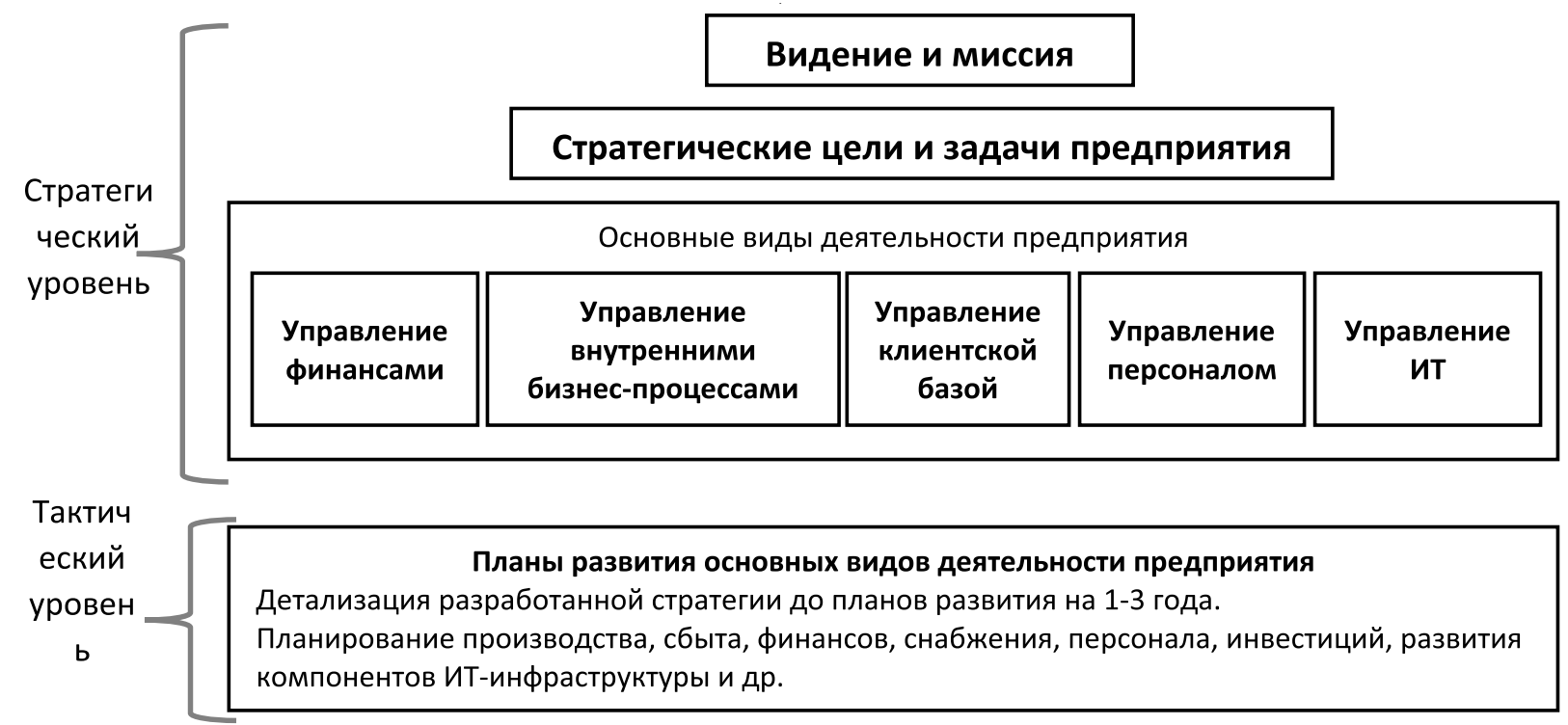

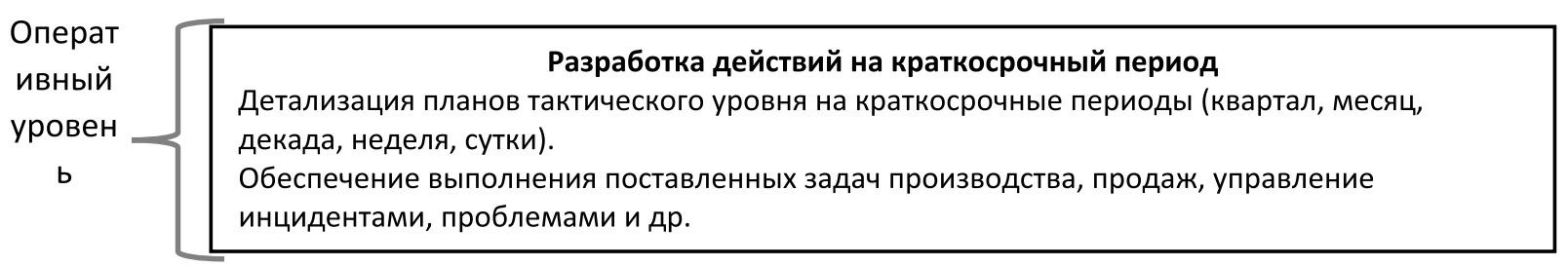

Рис. 2. Управление ИТ как стратегическая задача развития предприятия

продуктов, проходят контроль и верификацию. Бизнес-процессы консолидации, бюджетирования настраиваются в системе, пользователям предоставляются возможности формирования финансовых планов для всех участников процесса с учетом разных горизонтов планирования и разных уровней детализации планов, поддерживая вариативный анализ и разработку альтернативных сценариев. В системе имеется конфигуратор для настройки бюджетной модели, которая доступна на уровне пользователя. Бюджеты формируются на верхнем уровне, включая бюджеты доходов и расходов, движения денежных средств, прогнозный баланс, и операционные бюджеты. Особое внимание уделено контролю выполнения регламентов финансового планирования, корректировки и мониторинга бюджетов и исполнительной дисциплины. План-фактный анализ имеет визуальное представление в виде дашбордов, диаграмм с возможностью агрегации и детализации по каждому рассматриваемому показателю [12].

В рассмотренных системах реализован проектный подход для решения задач. Для того, чтобы достичь поставленных целей нужно разработать план для их достижения, определив значения показателей, по которым можно судить о степени достижения целей на стратегическом, тактическом и оперативном уровне.

\section{Возможности автоматизации управ^ения информационными технологиями на базе СРМ-систем}

Сегодня на рынке представлено большое количество программных продуктов, поддерживающих управление информационными технологиями и ИТ-инфраструктурой. Известен класс информационных систем оперативного управления ИТ и сетевой инфраструктурой - IT Operations Management, ITOM. Данные программные продукты позволяют автоматизировать различные составляющие ИТ-инфраструктуры, включая управление ИТ-сервисами, центром обработки данных и локальной сетью. Благодаря использованию систем данного класса появляется возможность консолидировать данные, получаемые из различных источников, осуществлять мониторинг всей ИТ-инфраструктуры, анализировать и контролировать различные параметры, такие как производительность, нагрузка, отслеживать действия пользователей. Это позволяет более эффективно осуществлять реагирование на инциденты и реализовывать проактивное управление ИТ. Программные продукты ITOM взаимодействуют с корпоративными системами на уровне API, обеспечивая коммуникации с ними.

Программное обеспечение ITOM поддерживает управление процессами согласно принятым стандар- 


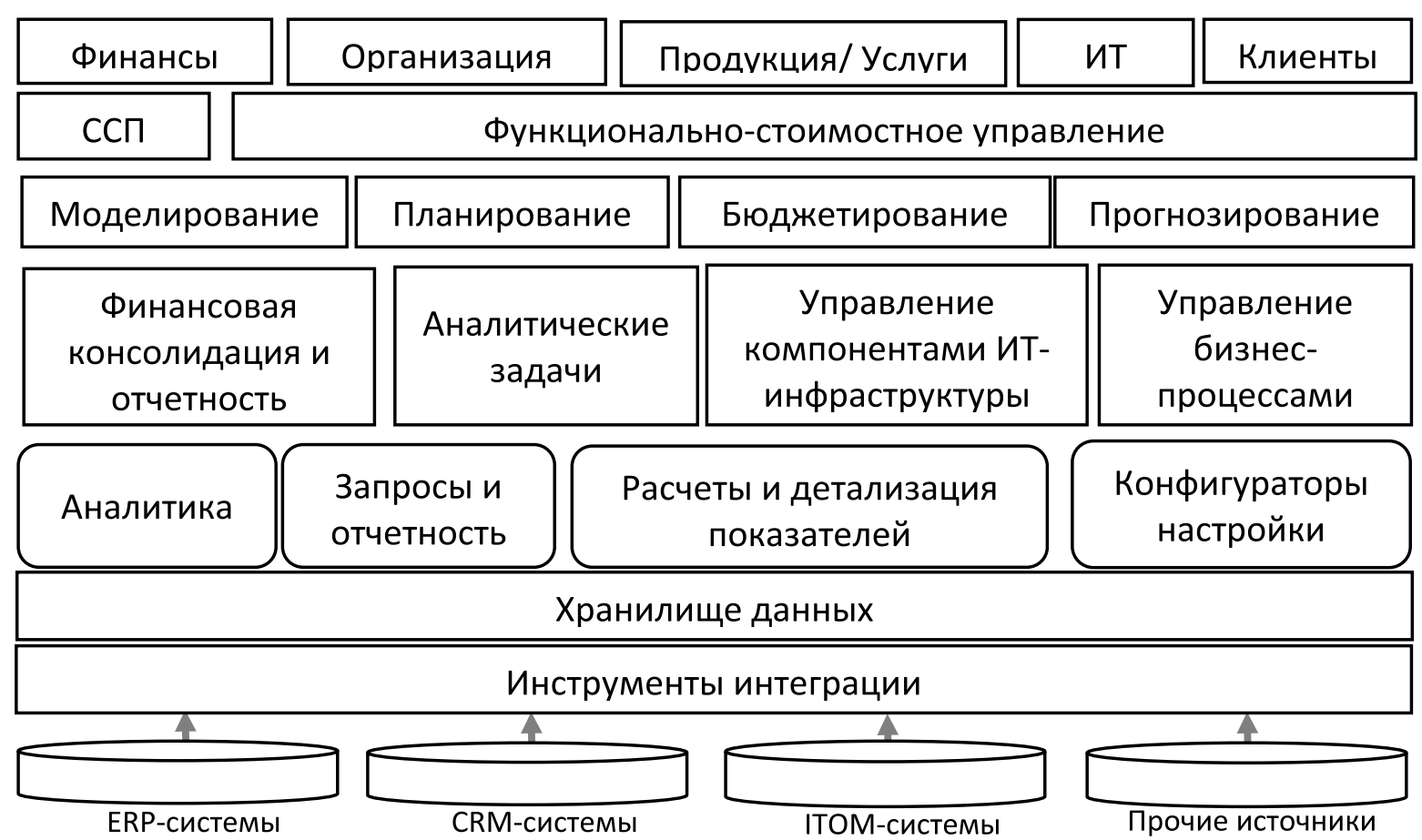

Рис. 3. Архитектура СРМ-системы

там и методикам управления информационными технологиями, автоматизируя управления активами, доступностью, производительностью, конфигурациями, сервисами Service Desk и Help Desk и др. Так, например, «1C: ITIL Управление информационными технологиями предприятия» соответствует ITIL V.3 и поддерживает процессы:

- предоставления услуг (управление уровнем и каталогом услуг, непрерывностью и доступностью услуг, мощностями, информационной безопасностью и др.);

- управления отношениями с потребителями и подрядчиками;

- управления знаниями, инцидентами, проблемами;

- управления конфигурациями, активами, изменениями;

- управления релизами и событиями;

- организации Service Desk [15].

Таким образом, поддержка управления информационными технологиями в данном программном продукте осуществляется на оперативном уровне, что, конечноже, соответствует принципам систем ITOM - оперативного управления ИТ и сетевой инфраструктурой.

Тем не менее современные стандарты и практики управления ИТ, специалисты в области планирования развития ИТ-инфраструктуры говорят о необходимости опираться на цели и задачи бизнеса при управлении информационными технологиями (рис. 2).

Автоматизировать управление информационными технологиями согласно принципам целевого управления можно на базе СРМ-систем. Поскольку системы управления эффективностью организаций реализуют проектный подход к управлению различными видами деятельности и, являясь «надстройкой сверху», интегрируются с системами транзакционного типа. Таким образом, в СРМ-системах осуществляется постановка целей и задач по управлению информационными технологиями, формируются планы с разным горизонтом реализации, назначаются ответственные, а ІТОМ-системы будут в этом случае являться поставщиками учетных данных (рис. 3).

На рис. 3 архитектура СРМ-системы включает платформу «бизнес-интеллекта» (Business Intelligence, BI), а также подсистемы, поддерживающие управление. С помощью подсистемы сбалансированной системы показателей ставятся цели и задачи развития предприятия традиционно в четырех составляющих: финансы, организация бизнеса (внутренние бизнес-процессы), продукты / услуги, клиенты, дополнительно могут быть определены цели и задачи управления информационными технологиями. Для мониторинга текущей ситуации СРМ-системы интегрируются с источниками корпоративной информации - ERP-, CRM-, ITOM-системами 
и другими источниками. Такой вариант автоматизации управления ИТ соответствует современным стандартам и представляет собой перспективное направление в сфере комплексного управления предприятием.

\section{Зак^ючение}

Обзор современных практик, методик и стандартов в области управления информационными технологиями показывает необходимость разработки планов развития ИТ-инфраструктуры предприятия на стратегическом уровне согласно целям и задачам бизнеса. Автомати- зировать эти задачи можно на базе систем класса СРМ. Благодаря реализуемому в системах данного класса проектному подходу ставятся цели и задачи для всех планируемых видов деятельности, включая управление информационными технологиями, разрабатываются бюджеты. Обратную связь с реализацией планов и фактическими данными СРМ-системы получают благодаря интеграции с системами транзакционного типа (ERP, CRM, ITOM и др.). Таким образом достигается поддержка полного цикла управления, включая планирование, организацию, контроль, учет и анализ достигнутых результатов.

\section{ЛИТЕРАТУРА}

1. Амириди Ю. В. 2019: Intersoft Lab: Российский СРМ-рынок достиг уровня зрелости // Tadviser [сайт]. URL: https://www.tadviser.ru/index.php/\%D0\%A1\%D1\% 82\%D0\%B0\%D1\%82\%D1\%8C\%D1\%8F: Corporate_Performance_Management\%2C_CPM_\%28\%D1\%80\%D1\%8B\%D0\%BD\%D0\%BE\%D0\%BA_\%D0\%A0\%D0 \%BE\%D1\%81\%D1\%81\%D0\%B8\%D0\%B8\%29 (дата обращения 11.01.2021).

2. Багинский К. Разработка ИТ-стратегии в крупных компаниях [сайт]. URL: https://mipt.ru/upload/ed3/f_fy3f-arpgxa6mq5q.pdf (дата 0бращения 11.01.2021).

3. Белов А. Автоматизация KPI-управления в среднем и крупном бизнесе [сайт].URL:https://solutions.1c.ru/catalog/kpi/materials (дата обращения 11.01.2021).

4. Горячев Р.А., Ильина 0.П. Информационные технологии управления эффективностью бизнеса — Business Performance Management. http://tvvlibrary. narod.ru/papers/2007/04.pdf (дата обращения 11.01.2021).

5. ГОСТ Р ИСО/МЭК 12207-2010 (полное наименование: ИСО/МЭК 12207-2010. Информационная технология. Системная и программная инженерия. Процессы жизненного цикла программных средств).

6. ГОСТ Р ИСО/МЭК 15288-2005 (полное наименование: ГОСТ Р ИСО/МЭК 15288-2005. Информационная технология. Системная инженерия. Процессы жизненного цикла систем).

7. ГОСТ 34.601-90 (полное наименование: ГОСТ 34.601-90. Информационная технология. Комплекс стандартов на автоматизированные системы. Автоматизированные системы. (тадии создания).

8. Китова О.В., Дьяконова Л. П. Информационно-аналитические технологии и сервисы в экономике и образовании // Инжиниринг предприятий и управление знаниями (ИП\&УЗ-2016): сборник научных трудов XIX научно-практической конференции. 26-27 апреля 2016 г. / под науч. ред. Ю. Ф. Тельнова. Москва: ФГБОУ В0 «РЭУ им. Г. В. Плеханова», 2016. — с. 30-36.

9. Конференция «Управление информационными технологиями в России 2020», [сайт]. URL: https://ict2go.ru/events/19315/ (дата обращения 11.01.2021).

10. 0бзор tAdviser CPM 2016. Системы повышения эффективности работы предприятия [сайт]. URL: http://www.tadviser.ru/index.php/CPM (дата обращения 29.12.2020).

11. Петрушов А. Запись вебинара «Отечественному рынку — отечественная ERP» [сайт]. URL: https://www.youtube.com/watch?v=3d1LLIRZyMs (дата обращения 11.01.2020).

12. CPM — Corporate Performance Management [сайт]. URL: https://www.tadviser.ru/index.php/\%D0\%A1\%D1\%82\%D0\%B0\%D1\%82\%D1\%8C\%D1\%8F: CPM_-_ Corporate_Performance_Management (дата обращения 03.01.2021).

13. The Value of Ongoing Performance Management. Brandon Hall Group, 2015 [сайт]. URL: http://www.iitd.ie/Portals/0/Knowledge\%20Centre/The-Value-ofOngoing-Performance-Manangement.pdf (дата обращения 11.01.2021).

14. C: ITIL Управление информационными технологиями предприятия [сайт]. URL: https://solutions.1c.ru/catalog/itil/features (дата 0бращения 11.01.2021).

(с) Одинцова Мария Александровна ( maryod@rambler.ru ).

Журнал «Современная наука: актуальные проблемы теории и практики» 